\title{
Capsule Commentary on Agana et al., a Novel Approach to Characterizing Readmission Patterns Following Hospitalization for Ambulatory Care-Sensitive Conditions
}

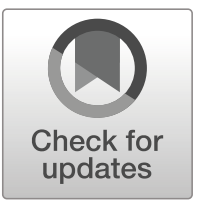

\author{
Aakash Bipin Gandhi, BPharm \\ Department of Pharmaceutical Health Services Research , University of Maryland School of Pharmacy, Baltimore, MD, USA.
}

J Gen Intern Med 35(4):1358

DOI: $10.1007 / \mathrm{s} 11606-020-05704-6$

(c) Society of General Internal Medicine 2020

$\mathrm{T}$ he retrospective observational study by Agana et al. ${ }^{1}$ utilized the Nationwide Readmission Database to describe patterns and determinants of readmissions among patients initially hospitalized for an ambulatory care-sensitive condition (ACSC). Factors associated with clustered (2 or more readmissions within 30 days of an index ACSC hospitalization) readmissions were also explored. Overall, younger age, a higher comorbidity burden, and having a chronic ACSC were associated with increased odds of experiencing a clustered readmission (compared no readmission) over the 270day follow-up period.

Due to the nature of the data, the findings of the study may be influenced by unmeasured confounding. For example, hospital admissions for ACSCs can be prevented through timely and effective outpatient care, often facilitated by primary care physicians (PCPs). ${ }^{2}$ However, the authors were unable to capture factors such as socioeconomic status or density of PCPs within the proximity of a patient's residence that could have influenced access to outpatient care. Additionally, behavioral factors such as non-adherence to medication regimens could not be captured. These factors, in turn, may have influenced readmission patterns. ${ }^{3-5}$ Nonetheless, the study helps identify key subgroups with a high propensity for readmissions following a hospitalization for an ACSC. These findings can help clinicians and health system administrators efficiently target intervention efforts.

The findings of this study can further benefit from future research. Data linkages with electronic health records can provide vital information on provider characteristics and patient behavioral factors that may influence readmission patterns. Linkages with local or county level data through patient residence ZIP codes can help illuminate the relationship between factors such as rural-urban residence and distance to health care resources that may affect readmission patterns. Hence, access to a comprehensive list of factors across multiple sources of data will help improve our understanding of reasons for ACSC readmissions and develop targeted intervention strategies to prevent the same.

Corresponding Author: Aakash Bipin Gandhi, BPharm; Department of Pharmaceutical Health Services Research University of Maryland School of Pharmacy, Baltimore, MD, USA (e-mail: aakash. gandhi@umaryland.edu).

\section{REFERENCES}

1. Agana DFG, Striley CW, Cook RL, Cruz-Almeida Y, Carek PJ, Salemi JL. A novel approach to characterizing readmission patterns following hospitalization for ambulatory care-sensitive conditions. J Gen Intern Med. https://doi.org/10.1007/s11606-020-05643-2.

2. Purdy S, Griffin T, Salisbury C, Sharp D. Ambulatory care sensitive conditions: terminology and disease coding need to be more specific to aid policy makers and clinicians. Public Health 2009; 123(2): 169-73

3. Kangovi S, Barg FK, Carter T, Long JA, Shannon R, Grande D. Understanding why patients of low socioeconomic status prefer hospitals over ambulatory care. Health Aff (Millwood). 2013;32(7):1196-203.

4. Continelli T, McGinnis S, Holmes T. The effect of local primary care physician supply on the utilization of preventive health services in the United States. Health Place 2010;16(5):942-51

5. Rosen OZ, Fridman R, Rosen BT, Shane R, Pevnick JM. Medication adherence as a predictor of 30-day hospital readmissions. Patient Prefer Adherence 2017;11:801.

Publisher's Note Springer Nature remains neutral with regard to jurisdictional claims in published maps and institutional affiliations.

Published online February 19, 2020 